\title{
Lateralized Theta Wave Connectivity and Language Performance in 2- to 5-Year-Old Children
}

\author{
Mitsuru Kikuchi, ${ }^{1,2}$ Kiyomi Shitamichi, ${ }^{2}$ Yuko Yoshimura, ${ }^{2,3}$ Sanae Ueno, ${ }^{2}$ Gerard B. Remijn, ${ }^{4}$ Tetsu Hirosawa, ${ }^{2}$ \\ Toshio Munesue, ${ }^{1}$ Tsunehisa Tsubokawa, ${ }^{5}$ Yasuhiro Haruta, ${ }^{6}$ Manabu 0i, ${ }^{1}$ Haruhiro Higashida, ${ }^{1}$ and Yoshio Minabe ${ }^{2}$ \\ ${ }^{1}$ Research Center for Child Mental Development, and ${ }^{2}$ Department of Psychiatry and Neurobiology, Graduate School of Medical Science, Kanazawa \\ University, Kanazawa 920-8641, Japan, ${ }^{3}$ Higher Brain Functions and Autism Research, Department of Child Development, United Graduate School of Child \\ Development, Kanazawa University and Hamamatsu University School of Medicine, Osaka University, Osaka 565-0871, Japan, ${ }^{4}$ International Education \\ Center, Kyushu University, Fukuoka, Japan 815-8545, 5Department of Anesthesiology, Graduate School of Medical Science, Kanazawa University, \\ Kanazawa 920-8641, Japan, and ${ }^{\circ}$ Department of MEG, Yokogawa Electric Corporation, Tokyo 180-8750, Japan
}

Recent neuroimaging studies support the view that a left-lateralized brain network is crucial for language development in children. However, no previous studies have demonstrated a clear link between lateralized brain functional network and language performance in preschool children. Magnetoencephalography $(\mathrm{MEG})$ is a noninvasive brain imaging technique and is a practical neuroimaging method for use in young children. MEG produces a reference-free signal, and is therefore an ideal tool to compute coherence between two distant cortical rhythms. In the present study, using a custom child-sized MEG system, we investigated brain networks while 78 right-handed preschool human children (32-64 months; 96\% were 3-4 years old) listened to stories with moving images. The results indicated that left dominance of parietotemporal coherence in theta band activity $(6-8 \mathrm{~Hz})$ was specifically correlated with higher performance of languagerelated tasks, whereas this laterality was not correlated with nonverbal cognitive performance, chronological age, or head circumference. Power analyses did not reveal any specific frequencies that contributed to higher language performance. Our results suggest that it is not the left dominance in theta oscillation per se, but the left-dominant phase-locked connectivity via theta oscillation that contributes to the development of language ability in young children.

\section{Introduction}

One of the most essential features of the human brain is the asymmetrical development of language functions between the two hemispheres. Even in the prenatal brain, anatomical asymmetries in language-related brain areas emerge as a function of brain development (Wada et al., 1975; Chi et al., 1977), suggesting that the bias for left hemisphere language lateralization is present quite early in development. Over the past decade, researchers have focused on how functional asymmetries relate to language development. Some studies have demonstrated a plausible link between brain functional asymmetries and language development in young children. Functional magnetic resonance imaging ( $\mathrm{fMRI}$ ) studies have demonstrated that left hemisphere dominance of language function may already be present in infants $<1$ year old (Dehaene-Lambertz et al., 2002; Peña et al., 2003). In older children ( $>5$ years old), not only left-lateralized

Received June 4, 2011; revised July 30, 2011; accepted Aug. 22, 2011.

Author contributions: M.K., H.H., and Y.M. designed research; M.K., K.S., Y.Y., S.U., G.B.R., T.M., T.T., and Y.M. performed research; M.K., K.S., Y.Y., S.U., G.B.R., T.H., Y.H., and M.O. analyzed data; M.K. wrote the paper.

This work was supported by the Hokuriku Innovation Cluster for Health Science (MEXT Program for Fostering Regional Innovation). We thank Gen Uehara (Kanazawa Institute of Technology) and Susumu Matsukura (Yokogawa Electric (orporation) for their technical support.

This article is freely available online through the J Neurosci Open Choice option.

Correspondence should be addressed to Mitsuru Kikuchi, Research Center for Child Mental Development, Kanazawa University, 13-1 Takara-machi, Kanazawa 920-8641, Japan. E-mail: mitsuru@zc4.so-net.ne.jp.

DOI:10.1523/JNEUROSCI.2785-11.2011

Copyright $\odot 2011$ the authors $\quad 0270-6474 / 11 / 3114984-05 \$ 15.00 / 0$ responses during language-related tasks (Balsamo et al., 2002; Ahmad et al., 2003), but also correlations between brain functional lateralization and language performance (Everts et al., 2009) have been reported. Previous fMRI studies, however, did not concentrate on the link between language performance and brain functional lateralization in healthy preschool children aged 1-4 years old, because this is a challenging age for brain imaging methods such as fMRI.

Coherent brain rhythms represent a core mechanism for sculpting temporal coordination of neural activity in the brainwide network (Wang, 2010). Brain rhythmic activity in one area is likely to be communicated to another and so generate temporal linkage in the rhythmicity in the two structures. For example, hippocampal theta rhythm is believed to play a crucial role in the formation and retrieval of episodic and spatial memory (Hasselmo, 2005), and theta rhythm was found to be enhanced in various neocortical sites during working memory in humans (Raghavachari et al., 2001; Meltzer et al., 2008). Coherent oscillations are thought to play a role in well timed coordination and communication between neural populations simultaneously engaged in cognitive processes (Wang, 2010). In the present study, we hypothesized that left lateralization of neuronal network connectivity is correlated with higher language performance in preschool children. For analysis of cortical network connectivity in 2- to 5-year-old children, we performed coherence analysis using a custom child-sized magnetoencephalogram (MEG) system (Kikuchi et al., 2010), because this is a noninvasive technique that 
A

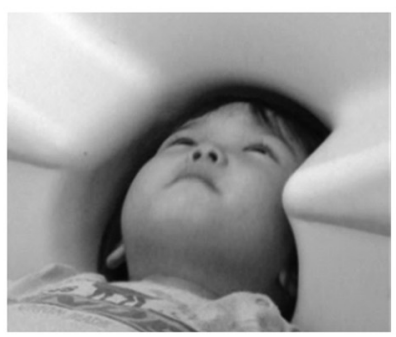

$\mathrm{C}$
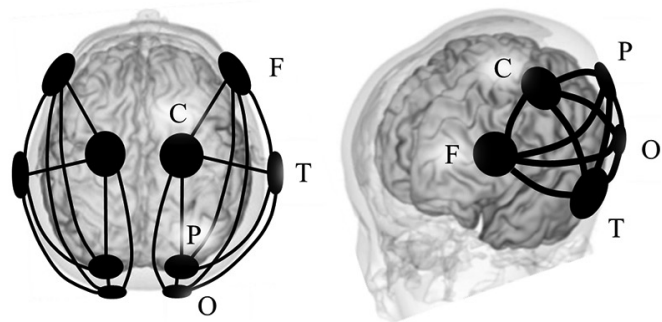

B

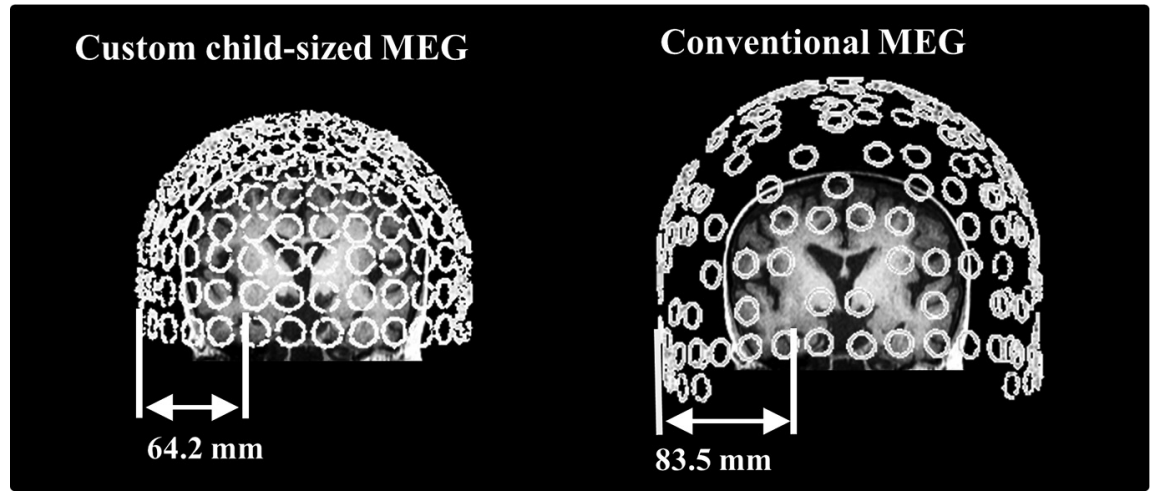

Figure 1. A, In the custom child-sized MEG system, the MEG sensors are as close to the whole head as possible for optima recording in young children. During MEG recording, the children lay supine on the bed and viewed video programs projected onto a screen. $\boldsymbol{B}$, Distances between MEG sensors (temporal area) and hippocampus. In 3- to 4-year-old children (left), sensors were close enough to the parahippocampal gyrus to record its activity, whereas in a conventional MEG system (right), the distance is too great to obtain a good signal from the parahippocampal gyrus. Open circles indicate MEG SQUID sensors (rear view). C, Schema of five selected sensors and 10 connections of interest (solid line) in each hemisphere.

provides measures of cortical neural activity on a millisecond time scale. Assessment of coherence can determine the degree of phase locking between activities recorded at different sensors, which have been used in clinical EEG studies (Wada et al., 1998; Kikuchi et al., 2002; Higashima et al., 2007). MEG produces a reference-free signal, and is therefore an ideal tool to compute coherence between two distant cortical rhythms. In addition, magnetic fields generated in the temporal lobe (e.g., auditory stimuli) tend to be reflected to sensors in the ipsilateral hemisphere, whereas electric potentials recorded by scalp EEG tend to spread to both hemispheres. This spatial property of MEG is particular advantageous in studies investigating the laterality of the cortical oscillations.

\section{Materials and Methods}

Seventy-eight right-handed children (36 boys, 42 girls) participated in the experiment. The participants had a mean age of 49.6 months (32-64). All subjects were native Japanese and had no previous or existing developmental, learning, or behavioral problems according to questionnaire information from their parents. All children participated in cognitive tasks and MEG measurements on two separate days. On both testing days, female staff members made contact with the children and played with them along with the parent(s)/caretaker(s). During MEG recording, one staff member (author Y.Y.) escorted each participant into the shielded room, which was decorated with colorful pictures of Japanese cartoon characters and mimicked an attractive vehicle adopted from an animation series popular with preschool children. During measurements, the staff member stayed in the shielded room, comforting and encouraging each participant to maintain a steady bodily position when necessary. Parent(s)/caretaker(s) could observe their child during measurements through a TV monitor. According to their evaluation, none of the participants endured high emotional tension or any other kind of discom- fort during measurements. Parents agreed to their child's participation in the study with full knowledge of the experimental nature of the research. Written informed consent was obtained before enrolment in the study. The Ethics Committee of Kanazawa University Hospital approved the methods and procedures, all of which were in accordance with the Declaration of Helsinki.

The Kaufman Assessment Battery for Children was applied in all children. The mean score on the mental processing scale was $99.90 \pm 13.50$ (mean $\pm \mathrm{SD})$ in all participants $(n=78)$, and the mean score on the achievement scale was $103.79 \pm 16.16$ in 76 participants (two participants were not available for assessment on the achievement scale because they were younger than 36 months). As we were especially interested in the relation between brain lateralization and development of language performance in children, languagerelated cognitive performance was calculated by adding the scores of the Expressive Vocabulary and Riddles subtests. In addition, nonverbal cognitive performance was calculated by adding the scores of the Face Recognition and Hand Movement subtests (Kaufman and Kaufman, 1983).

MEG data were recorded with a 151-channel superconducting quantum interference device (SQUID) whole-head coaxial gradiometer MEG system for children (PQ 1151R; Yokogawa/KIT) in a magnetically shielded room (Daido Steel). MEG data were acquired with a sampling rate of $1000 \mathrm{~Hz}$ and filtered with a $200 \mathrm{~Hz}$ low-pass filter. One MEG session lasted for $6 \mathrm{~min}$. The experiment consisted of one to eight sessions with $\sim 5 \mathrm{~min}$ breaks in between. The number of sessions depended on the participant's will (i.e., we finished experiments when participants did not want to continue). In the present study, we used data from the first session from each participant. During MEG recording, children lay supine on the bed and viewed a video program projected onto a screen. We confirmed the position of the head to be in the center of the MEG helmet by measuring the magnetic fields after passing currents through coils that were attached at three locations on the head surface as fiduciary points with respect to the landmarks (bilateral mastoid processes and nasion). Before recording, we prepared a number of video programs with stories especially attractive to young children. The video program was selected according to the preference of each participant. Sound of the narrations was carried to participants binaurally through a tube in front of the subject from speakers placed outside the shielded room. Before recording, we ensured that they were content with the video program selected.

Off-line analysis of the MEG data was performed with Brain Vision analyzer (Brain Products) and Matlab (MathWorks). MEG data were resampled at $500 \mathrm{~Hz}$. Data were segmented for $2.5 \mathrm{~s}$. Selection of artifactfree segments was based on visual inspection. The process of eliminating contaminated data was performed blind to personal data. At least 15 artifact-free segments ( $35 \mathrm{~s}$ period) were accepted for each subject. MEG spectra were calculated with the fast Fourier transform with a spectral resolution of $0.244 \mathrm{~Hz}$. In this study, 10 intrahemispheric coherences for each hemisphere were measured between the following five regions of interest: frontal $(\mathrm{F})$, central $(\mathrm{C})$, temporal $(\mathrm{T})$, parietal $(\mathrm{P})$, and occipital (O) areas (Fig. 1C). Before the off-line analysis, selection of sensors corresponding to these brain areas was done based on the following algorithm. First, using MRI brain anatomical images in three young children, we calculated the distance between sensor and hippocampus or posterior cingulate cortex (PCC) for 151 sensors. Then, in each hemisphere, we identified sensors of nearest distance to hippocampus or PCC. These sensors were located close to the $\mathrm{T}$ or $\mathrm{P}$ area, respectively. Next, we 
selected sensors corresponding to F, C, and $\mathrm{O}$ areas based on the following requirements: (1) intersensor distances between T-F, T-C, or T-O were almost the same as those between T-P (i.e., within a range of $100-$ $120 \mathrm{~mm}$ ); (2) the sensor corresponding to the F area was selected among front row sensors of the MEG device; (3) the sensor corresponding to the $\mathrm{C}$ area was selected from a row of sensors midway between the sensors corresponding to the F and P areas; and (4) the sensor corresponding to the $\mathrm{O}$ area was selected so that the sensor corresponding to the $\mathrm{P}$ area was located in midway between the sensors corresponding to the $\mathrm{C}$ and $\mathrm{O}$ areas. Eventually, the sensors corresponding to the $\mathrm{F}$ and $\mathrm{O}$ areas were located close to the dorsal prefrontal area and occipital area, respectively. Coherence and relative power were banded into the following eight bands: delta $(0.7-3.9 \mathrm{~Hz})$, theta-1 $(4.2-5.9 \mathrm{~Hz})$, theta-2 $(6.4-7.8 \mathrm{~Hz}), \alpha-1$ (8.3-9.8 Hz), $\alpha-2(10.0-12.0 \mathrm{~Hz}), \beta-1(12.2-19.8 \mathrm{~Hz}), \beta-2(20.0-29.8$ $\mathrm{Hz}$ ), and gamma $(30.0-57.9 \mathrm{~Hz})$. Laterality indices (LIs) were calculated for each coherence and each relative power using the following formula: $\mathrm{LI}=(\mathrm{L}-\mathrm{R}) /(\mathrm{L}+\mathrm{R})$, where $\mathrm{L}$ and $\mathrm{R}$ are the left and right hemispheres, respectively.

Pearson's correlation was used to find significant correlations between physiological measures (i.e., intrahemispheric coherence or relative power values in both hemispheres and their LIs) and cognitive performance (or chronological age). Our alpha level was adjusted to $0.05 / 80=$ $0.0006(r>0.379)$ for coherence analysis and to $0.05 / 40=0.0012(r>$ 0.365 ) for relative power analysis, because of the multiple comparisons in 10 interhemispheric pairs in coherence analyses (or five hemispheric sensors in power analyses) for the eight frequency bands. As a complementary approach, an alpha level of 0.05 was also used at the risk of a type I error to explore correlations between physiological measures and language-related cognitive performance.

\section{Results}

\section{Intrahemispheric coherence}

There were no significant correlations between intrahemispheric coherences and cognitive performance or chronological age in either hemisphere ( $p>0.0006$; Fig. $2 B, C)$.

\section{LI of intrahemispheric coherence}

There was a significant positive correlation between LI of theta-2 band coherence (parietotemporal network) and language-related cognitive performance $(r=0.390, p<0.0006)$ (Figs. $2 A, 3)$. This LI showed no significant correlation with nonverbal cognitive performance, chronological age (Fig. 3), or head circumference. There were no significant correlations between LI of any other coherence and cognitive performance or chronological age. To evaluate the existence of a possible gender and/or age effect on the significant relationship found in LI of theta-2 band coherence $(\mathrm{P}-\mathrm{T})$, we used multiple linear regression to predict the LI (i.e., dependent variable) using language-related cognitive performance, age, and gender as predictors (i.e., three independent variables). The significance level was set at $p<0.05$. In the multiple regression model, language-related cognitive performance was the significant predictor of the LI $(n=78, \beta=0.362, p<$ $0.05)$, whereas age $(n=78, \beta=0.002, p>0.05)$ and gender $(n=$ $78, \beta=-0.175, p>0.05)$ did not reach statistical significance.

\section{Relative power value}

There was a significant negative correlation between relative power value in delta band in right $\mathrm{P}$ area and chronological age $(r=-0.398, p<0.0012)$. There were no significant correlations between any other relative power value and cognitive performance or chronological age in either hemisphere $(p>0.0012)$.

\section{LI of relative power}

There were no significant correlations between LI of any region and cognitive performance, chronological age, or head circumference $(p>0.0012)$.
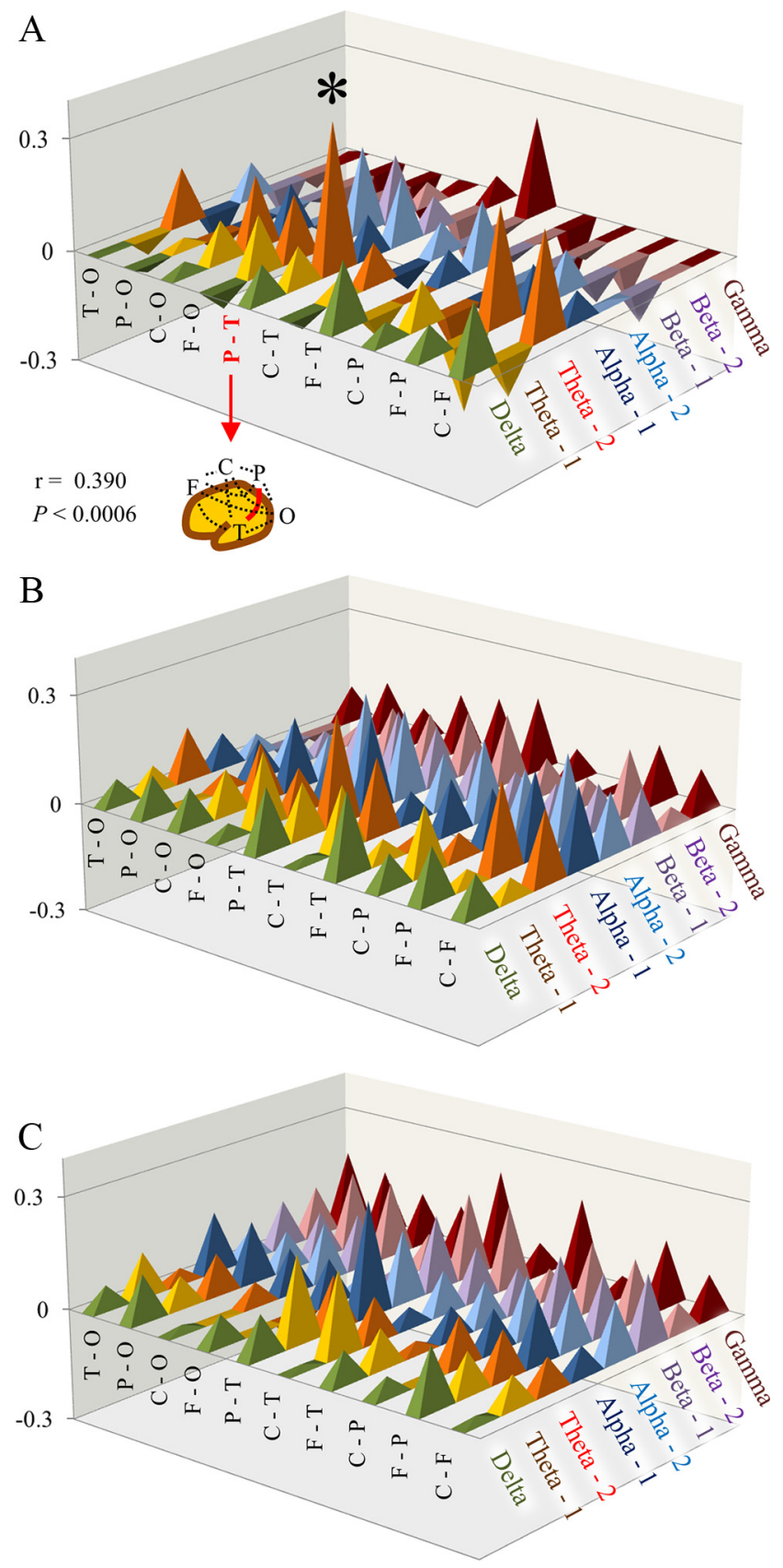

Figure 2. A, Correlation coefficients between laterality index of intrahemispheric coherences and language-related cognitive performance. Note that a significant correlation was found in parietotemporal connectivity in theta-2 $(r=0.390, p<0.0006)$. $\boldsymbol{B}, \boldsymbol{C}$, Correlation coefficients between intrahemispheric coherences and language-related cognitive performance in the left $(\boldsymbol{B})$ or right $(\boldsymbol{C})$ hemisphere. There were no significant correlations between intrahemispheric coherences and cognitive performance in either hemispheres $(p \geq 0.0006)$. ${ }^{*} p<0.0006$.

If we used an alpha level of 0.05 at the risk of a type I error, some significant correlations arose between physiological measures and language-related cognitive performance. If we focused on the theta- 2 band oscillation, the following result emerged in addition to the results above. There were positive correlations in LIs of coherence ( $\mathrm{F}-\mathrm{P}$ and C-F; $r=0.295$ and 0.269 , respectively) and in the left coherence values (P-T and F-P; $r=0.295$ and 0.234 , respectively). There was a negative correlation in the right relative power value ( $\mathrm{C}$ area; $r=-0.240$ ). 

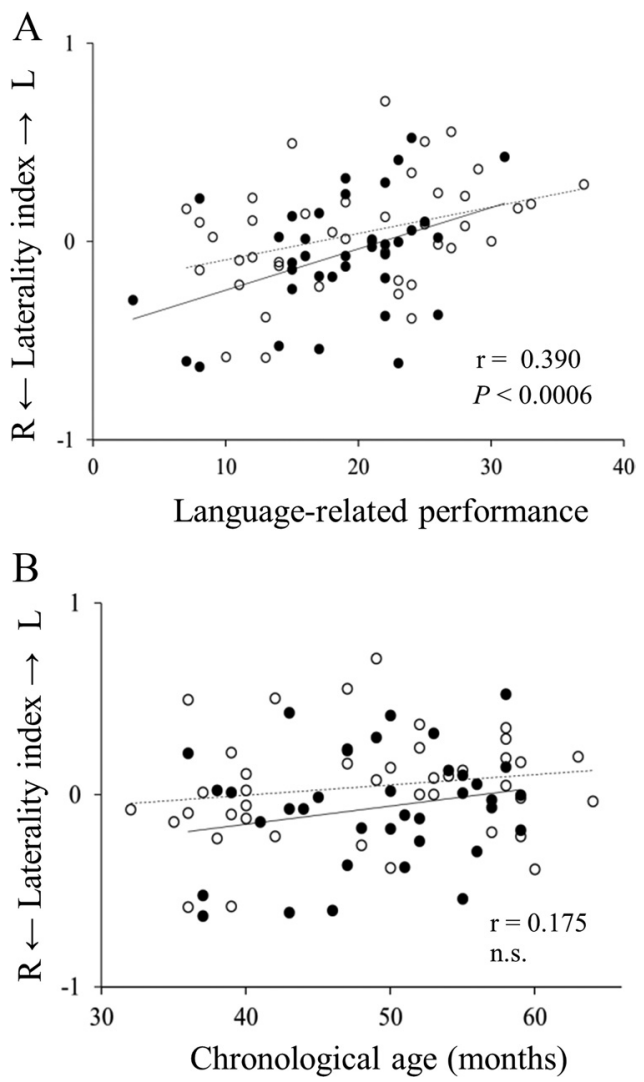

Figure 3. $\quad A, B$, Scatter plot of laterality index of theta- 2 band coherence (parietotemporal connectivity) and language-related cognitive performance $(r=0.390, p<0.0006 ; \boldsymbol{A})$, and chronological age $(r=0.175$, n.s.; $\boldsymbol{B})$. $\bigcirc$, Boys $(n=36)$;, girls $(n=42)$. Solid line, Regression line for boys; broken line; regression line for girls. L, left; $R$, right; n.s., not significant.

\section{Discussion}

In terms of brain functional connectivity in 2- to 5-year-old children, this is the first report demonstrating the advantages of left-lateralized connectivity via theta oscillation for higher performance in semantic processing. A recent study demonstrated this left lateralization in intrahemispheric hemodynamic connectivity using fMRI in older children (5-18 years old) (Karunanayaka et al., 2007). This was the only previous study dealing with functional connectivity in children; our results are consistent with theirs. Furthermore, our results are in agreement with many recent anatomical studies in healthy adult and child populations. Recent anatomical studies using diffusion tensor MRI demonstrated that left lateralization in the superior longitudinal fasciculus/arcuate fasciculus is correlated with languagerelated performance in both healthy adults (Catani et al., 2007) and in 5- to 13-year-old children (Lebel and Beaulieu, 2009), while this language pathway in the left hemisphere appeared to be functionally insufficient in 5- to 8-year-old children (Brauer et al., 2011). In addition, in healthy infants aged $0-3$ years old, a recent volumetric study suggested the importance of relationships between progression of myelin deposition in the left hemispheric white matter and children's vocabulary acquisition (Pujol et al., 2006). Most of these previous anatomical studies focused on the auditory-language pathway from Wernicke's area to Broca's area (e.g., superior longitudinal fasciculus/arcuate fasciculus) and supported the view that the left-lateralized brain network is crucial for the acquisition of linguistic abilities not only in adults but also in developing young children. In the present study, we demonstrated that left dominance of parietotemporal coherence in the theta band is correlated with higher performance on language-related tasks, whereas this laterality is not correlated with chronological age or head circumference. The relatively small age range of the children (32-64 months; $96 \%$ were 3-4 years old) in the present study may have contributed to a reduction in the brain connectivity variation due to age differences. We failed to demonstrate a significant result for frontotemporal coherence. This negative result might be attributed to the position of the sensor corresponding to the frontal area. In the present study, to achieve sparse alignment of MEG sensors, the sensor in frontal area was located close to the dorsal prefrontal area (rather than Broca's area).

Given that the processing of language information involves interactions between multiple regions of the brain, maturation of the long-distance network should be crucial for language development. In fact, slower (theta) oscillation has been suggested to be involved in long-distance networks associated with top-down processing (Siegel et al., 2000; von Stein and Sarnthein, 2000; von Stein et al., 2000) or with working memory and attention (Sarnthein et al., 1998). In addition, phasic increases of theta activity have been reported during language comprehension tasks (Bastiaansen et al., 2005; Bastiaansen and Hagoort, 2006; Hald et al., 2006). Anomalous asymmetry of theta EEG activity during linguistic tasks was reported in the case of developmental dyslexia (children $\sim 10$ years old) (Spironelli et al., 2008). These previous studies and our results support the suggestion that brain activities in language-related brain regions generate left-lateralized temporal linkages via theta oscillations, which contribute to normal language development.

The sensors (axial gradiometers) of this child-MEG system originally had the potential to record brain oscillation even from deep brain structures (Kimura et al., 2008), and the combination of young children (i.e., thin skull) and custom-sized MEG system (i.e., close sensors) achieved a shorter distance between sensors and deeper brain structures, such as the hippocampal formation. Before this research, using MRI brain images in three young children, we estimated the distance between the sensors selected for the temporal lobes and hippocampus. The mean distance was $64.2 \mathrm{~mm}$ for the custom child-sized MEG system, compared with $84.5 \mathrm{~mm}$ for a conventional MEG system (PQ 1160C; Yokogawa Electric) (Fig. 1 $A, B$ ). As a result, in the medial temporal cortex, the signal-to-noise ratio in the custom child-sized MEG system was $\sim 1.8$-fold higher. Although caution must be exercised in drawing any definitive conclusions, because the oscillations of deep brain structures cannot be discriminated from those of shallow brain structures in our analysis, there is no doubt that the magnetic signals recorded by the sensors corresponding to temporal areas contained signals from medial temporal structures (e.g., hippocampal formation). Furthermore, a recent study using intracerebral EEG in patients with epilepsy demonstrated that successful recall in the auditory verbal learning test was associated with the enhancement of temporal synchronization of the theta rhythms within a cerebral network including the hippocampus, amygdala, and temporal-occipital neocortex (Babiloni et al., 2009). Thus, it is possible that brain rhythmic activities in medial temporal structures generate temporal linkages over the temporal or parietal neocortex via theta rhythmicity during the learning process, and that left lateralization in such linkages may contribute to the higher language performances, as observed in the present study.

One critical limitation should be taken into account when considering the results of coherence analysis, as performed in the 
present study. Assessment of coherence (i.e., phase correlation extracted from the Fourier transform) determines the degree of phase lag consistencies between activities recorded at different sensors. However, coherence between a pair of sensors can increase not only because there are two distinct sources, providing activity that has become more phase-locked, but also because a single source generates a signal that reaches both sensors. In our study, to reduce the latter possibility, we used sparse alignment of MEG sensors (e.g., the distance between temporal and parietal regions was $120 \mathrm{~mm}$ in each hemisphere). Given that magnetic field strength diminishes approximately with the square of the distance from the source, there must have been a strong source for theta oscillation if only one source influenced the coherence between the temporal and parietal area. However, in the present study, power analyses in temporal and parietal areas failed to demonstrate any significant relationships with language-related cognitive performance. Therefore, it seems plausible that the higher language performance in children is indeed correlated with the left-dominant phase-locking between two distinct brain regions (i.e., temporal and parietal).

\section{Notes}

Supplemental material for this article is available at http://blog-imgs50.fc2.com/m/i/t/mitsurukikuchi/20110730164911833.htm. Table S1 shows correlation coefficients between laterality index of intrahemispheric coherences and language-related cognitive performance. Table S2 shows correlation coefficients between intrahemispheric coherence (in left or right hemisphere) and language-related cognitive performance. Table S3 shows correlation coefficients between laterality index of power value and language-related cognitive performance. Table S4 shows correlation coefficients between relative power value and language-related cognitive performance. This material has not been peer reviewed.

\section{References}

Ahmad Z, Balsamo LM, Sachs BC, Xu B, Gaillard WD (2003) Auditory comprehension of language in young children: neural networks identified with fMRI. Neurology 60:1598-1605.

Babiloni C, Vecchio F, Mirabella G, Buttiglione M, Sebastiano F, Picardi A, Di Gennaro G, Quarato PP, Grammaldo LG, Buffo P, Esposito V, Manfredi M, Cantore G, Eusebi F (2009) Hippocampal, amygdala, and neocortical synchronization of theta rhythms is related to an immediate recall during Rey auditory verbal learning test. Hum Brain Mapp 30:2077-2089.

Balsamo LM, Xu B, Grandin CB, Petrella JR, Braniecki SH, Elliott TK, Gaillard WD (2002) A functional magnetic resonance imaging study of left hemisphere language dominance in children. Arch Neurol 59:1168-1174.

Bastiaansen M, Hagoort P (2006) Oscillatory neuronal dynamics during language comprehension. Prog Brain Res 159:179-196.

Bastiaansen MC, van der Linden M, Ter Keurs M, Dijkstra T, Hagoort P (2005) Theta responses are involved in lexical-semantic retrieval during language processing. J Cogn Neurosci 17:530-541.

Brauer J, Anwander A, Friederici AD (2011) Neuroanatomical prerequisites for language functions in the maturing brain. Cereb Cortex 21:459-466.

Catani M, Allin MP, Husain M, Pugliese L, Mesulam MM, Murray RM, Jones DK (2007) Symmetries in human brain language pathways correlate with verbal recall. Proc Natl Acad Sci U S A 104:17163-17168.

Chi JG, Dooling EC, Gilles FH (1977) Left-right asymmetries of the temporal speech areas of the human fetus. Arch Neurol 34:346-348.

Dehaene-Lambertz G, Dehaene S, Hertz-Pannier L (2002) Functional neuroimaging of speech perception in infants. Science 298:2013-2015.

Everts R, Lidzba K, Wilke M, Kiefer C, Mordasini M, Schroth G, Perrig W, Steinlin M (2009) Strengthening of laterality of verbal and visuospatial functions during childhood and adolescence. Hum Brain Mapp 30:473-483.
Hald LA, Bastiaansen MC, Hagoort P (2006) EEG theta and gamma responses to semantic violations in online sentence processing. Brain Lang 96:90-105.

Hasselmo ME (2005) What is the function of hippocampal theta rhythm? Linking behavioral data to phasic properties of field potential and unit recording data. Hippocampus 15:936-949.

Higashima M, Takeda T, Kikuchi M, Nagasawa T, Hirao N, Oka T, Nakamura M, Koshino Y (2007) State-dependent changes in intrahemispheric EEG coherence for patients with acute exacerbation of schizophrenia. Psychiatry Res 149:41-47.

Karunanayaka PR, Holland SK, Schmithorst VJ, Solodkin A, Chen EE, Szaflarski JP, Plante E (2007) Age-related connectivity changes in fMRI data from children listening to stories. Neuroimage 34:349-360.

Kaufman A, Kaufman N (1983) Kaufman Assessment Battery for Children: administration and scoring manual. Circle Pines, MN: American Guidance Service.

Kikuchi M, Wada Y, Takeda T, Oe H, Hashimoto T, Koshino Y (2002) EEG harmonic responses to photic stimulation in normal aging and Alzheimer's disease: differences in interhemispheric coherence. Clin Neurophysiol 113:1045-1051.

Kikuchi M, Shitamichi K, Ueno S, Yoshimura Y, Remijn GB, Nagao K, Munesue $\mathrm{T}$, Iiyama $\mathrm{K}$, Tsubokawa $\mathrm{T}$, Haruta $\mathrm{Y}$, Inoue $\mathrm{Y}$, Watanabe $\mathrm{K}$, Hashimoto T, Higashida H, Minabe Y (2010) Neurovascular coupling in the human somatosensory cortex: a single trial study. Neuroreport $21: 1106-1110$

Kimura T, Ozaki I, Hashimoto I (2008) Impulse propagation along thalamocortical fibers can be detected magnetically outside the human brain. J Neurosci 28:12535-12538.

Lebel C, Beaulieu C (2009) Lateralization of the arcuate fasciculus from childhood to adulthood and its relation to cognitive abilities in children. Hum Brain Mapp 30:3563-3573.

Meltzer JA, Zaveri HP, Goncharova II, Distasio MM, Papademetris X, Spencer SS, Spencer DD, Constable RT (2008) Effects of working memory load on oscillatory power in human intracranial EEG. Cereb Cortex 18:1843-1855.

Peña M, Maki A, Kovacić D, Dehaene-Lambertz G, Koizumi H, Bouquet F, Mehler J (2003) Sounds and silence: an optical topography study of language recognition at birth. Proc Natl Acad Sci U S A 100:11702-11705.

Pujol J, Soriano-Mas C, Ortiz H, Sebastián-Gallés N, Losilla JM, Deus J (2006) Myelination of language-related areas in the developing brain. Neurology 66:339-343.

Raghavachari S, Kahana MJ, Rizzuto DS, Caplan JB, Kirschen MP, Bourgeois B, Madsen JR, Lisman JE (2001) Gating of human theta oscillations by a working memory task. J Neurosci 21:3175-3183.

Sarnthein J, Petsche H, Rappelsberger P, Shaw GL, von Stein A (1998) Synchronization between prefrontal and posterior association cortex during human working memory. Proc Natl Acad Sci U S A 95:7092-7096.

Siegel M, Körding KP, König P (2000) Integrating top-down and bottom-up sensory processing by somato-dendritic interactions. J Comput Neurosci 8:161-173.

Spironelli C, Penolazzi B, Angrilli A (2008) Dysfunctional hemispheric asymmetry of theta and beta EEG activity during linguistic tasks in developmental dyslexia. Biol Psychol 77:123-131.

von Stein A, Sarnthein J (2000) Different frequencies for different scales of cortical integration: from local gamma to long range alpha/theta synchronization. Int J Psychophysiol 38:301-313.

von Stein A, Chiang C, König P (2000) Top-down processing mediated by interareal synchronization. Proc Natl Acad Sci U S A 97:14748-14753.

Wada JA, Clarke R, Hamm A (1975) Cerebral hemispheric asymmetry in humans: cortical speech zones in 100 adults and 100 infant brains. Arch Neurol 32:239-246.

Wada Y, Nanbu Y, Kikuchi M, Koshino Y, Hashimoto T (1998) Aberrant functional organization in schizophrenia: analysis of EEG coherence during rest and photic stimulation in drug-naive patients. Neuropsychobiology 38:63-69.

Wang XJ (2010) Neurophysiological and computational principles of cortical rhythms in cognition. Physiol Rev 90:1195-1268. 